Original Research Paper

\title{
Integrated Modeling Framework for Highway Traffic Pollution Estimation and Dispersion
}

\author{
${ }^{1}$ Shu Yang, ${ }^{1}$ Yao-Jan Wu and ${ }^{2}$ John Woolschlager \\ ${ }^{I}$ Department of Civil Engineering and Engineering Mechanic, The University of Arizona, Tucson, Arizona, USA \\ ${ }^{2}$ Center for Sustainability, Saint Louis University, Saint Louis, Missouri, USA
}

Article history

Received: 17-01-2016

Revised: 10-05-2016

Accepted: 11-05-2016

Corresponding Author:

Shu Yang

Department of Civil

Engineering and Engineering

Mechanic, The University of

Arizona, Tucson, Arizona,

USA

Email: shuyang@email.arizona.edu

\begin{abstract}
Air quality is of great concern to the public. Airborne pollutants, such as carbon monoxide (CO), carbon dioxide $\left(\mathrm{CO}_{2}\right)$ and Particulate Matter (PM), negatively impact people's living conditions. In particular, vehicular exhaust is a major source of these pollutants. Extensive previous research has contributed to modeling traffic pollutant emission. Popular approaches include computational simulation-based methods, which have been dominant in studies related to traffic emissions. However, simulation-based methods may not reflect changes in traffic and environmental factors in a real world situation. In order to address this and other limitations, our study employs real world traffic, meteorological and geographical data. In order to fully utilize this data and apply it to traffic pollutant emission and dispersion modeling, this study proposes a framework to integrate a traffic emission model, dispersion model and multi-source public data to best estimate traffic emissions and dispersion. The framework is based on the concept that traffic sensors + meteorology sensors $=$ emissions sensors. The results from case studies show that the concept is feasible and that air pollutants can be estimated over a large area. Sensitivity analyses on atmosphere stability and surface roughness showed not only the effectiveness of the proposed framework, but also demonstrated potential applications for inter-disciplinary research including public health and land-use planning.
\end{abstract}

Keywords: Vehicle Pollutant Emission, Pollutant Dispersion, Data Integration, Geographic Information System (GIS) and Visualization

\section{Introduction}

Air quality is of great concern to the public and managing agencies. Airborne pollutants, such as carbon monoxide (CO) and Particulate Matter (PM), negatively impact people's living conditions. For example, nitrogen oxides (NOx) and PM, which are common pollutants, may damage the respiratory system (e.g., causing fluid build-up in lungs and shortness of breath). Additionally, $\mathrm{CO}_{2}$ from vehicle emissions may lead to climate change and associated environmental problems including rising sea levels. Vehicular exhaust is one of the major sources of these pollutants (Raaschou-Nielsen et al., 2001; CDPH, 2014) and commonly considered by city planners and transportation planners as one of the major environmental factors to consider when conducting short- and long-term development planning.
A direct approach to measuring the air pollutant concentration produced by traffic is to use emission monitoring sensors. However, this approach may not be appropriate for monitoring large areas due to the cost of sensor installation and usage. Hence, ample research has contributed to modeling traffic pollutant emissions instead. During early research stages, traffic volume information is the major variable for estimating traffic pollutant emissions. Aldrin and Haff (2005) proposed a statistical model to quantify the empirical relationships between traffic volume, meteorology data and the level of pollution. Xia and Shao (2005) estimated traffic pollution on a road network where traffic flow was simulated by their proposed Lagrangian model. A sensitivity analysis estimating the amount of air pollution was conducted in different traffic flow scenarios. Berkowicz et al. (2006) used the COPERT model to estimate traffic emissions by using the daily 
average traffic volume. Their traffic volume data was collected from both automated and manual traffic detectors. Berkowicz et al. (2006) pointed out that travel speed information, although not considered in their study, would significantly affect traffic emission estimation in real-world situations. In recent years, traffic speed information has received growing attention. Hatzopoulou and Miller (2010) used an activity-based travel demand model to generate vehicle activities, instead of conventional traffic flow simulation models without speed information. Smit et al. (2008) estimated traffic emission inventories by taking advantage of average speed distributions. The required traffic data, including speed data and traffic volume, was generated by a macroscopic dynamic traffic assignment model. Lee et al. (2012) used Origin and Destination (O-D) demand inputs to produce detailed vehicle movement information over a network through a microscopic traffic simulation model (TransModeler). A combination of a traffic emission estimation model (MOVES) and a regional air pollutant dispersion model was then used to calculate traffic emissions and dispersion. Ishaque and Noland (2008) analyzed the level of pedestrian exposure to traffic emissions. A micro-simulation model was utilized to produce the details of vehicle and pedestrian movements, including individual travel speed. The model output was used as an input of traffic emissions estimation.

Previous research (Aldrin and Haff, 2005; Berkowicz et al., 2006; Jin and $\mathrm{Fu}, 2005$ ) has primarily focused on modeling either traffic emissions or emissions dispersion separately. Traffic emission data is one of the major inputs for air pollutant dispersion analysis. Dispersion analysis results could help better understand the impact of emissions on public health across geographical regions. Hence, an increasing amount of research (Hatzopoulou and Miller, 2010; Amirjamshidi et al., 2013) has integrated both traffic emission and dispersion analyses to investigate their chain reaction. Amirjamshidi et al. (2013) incorporated regional travel demand models and a microscopic traffic simulation model to estimate necessary traffic-related data. The output was streamed into a vehicle emission model to produce traffic emission data; then an air pollutant dispersion model was applied to calculate dispersion using both the traffic emission data and the estimated predominant wind direction that was obtained from a meteorological model. With the objective of analyzing health impacts caused by heavy trucks, Lee et al. (2012) combined a microscopic traffic simulation model with an air pollutant dispersion model to assess air quality and evaluate the impact of traffic emissions on public health. Hourly meteorological data was purchased to estimate the air pollutant dispersion.
The limitations of previous studies are summarized below:

Computational traffic simulation modeling has frequently been used in traffic emission-related studies (Xia and Shao, 2005; Amirjamshidi et al., 2013). O-D data is the main input for estimating traffic emissions at a network or regional level. Travel demand models, such as conventional four-stage models and activity-based models, use O-D data to simulate detailed vehicle movements over networks. On the other hand, microscopic traffic models are typically used to produce vehicle movement details when local traffic volume information is unavailable.

With the increasing coverage of traffic sensors on roadways and the growing availability of personal device data, Intelligent Transportation Systems (ITS) have become one of the major traffic data sources. However, ITS data has been used primarily for traffic operation and management (Wu et al., 2011; Ma et al., 2011). Li et al. (2009) utilized GPS data from transit buses to estimate the emissions caused by buses. Jeng et al. (2013) employed inductive loop detector data to estimate traffic emissions on a freeway corridor. These ITS data-based research projects specifically focused more on traffic emission modeling instead of proposing an integrated emission and dispersion modeling framework.

Meteorological data was not widely available to previous researchers. Amirjamshidi et al. (2013) and Berkowicz et al. (2006) had to estimate meteorological data instead of collecting it in the field. Therefore, meteorological data is generally assigned fixed values and unable to reflect the changing weather condition over time. Jamriska et al. (2008) pointed out that meteorological information, especially wind direction, greatly affected the model results for traffic emission generation and dispersion.

Finally, few studies on evaluating traffic pollution have considered atmospheric stability conditions. Atmospheric stability is an essential component in air pollutant dispersion, because atmospheric conditions may change greatly during a short time period.

Overall, few studies have developed an integrated framework that handles both real-world traffic data and meteorological data to estimate traffic pollutant emissions and dispersion. The main challenges include data availability and modeling framework design. The most recent and also the most relevant study for highways were presented by Samaranayake et al., (2014). The authors proposed a novel air pollution estimation method that modeled traffic conditions, traffic-induced air pollution emissions and pollution dispersion. Their study focused on developing a prototype system rather than on applying it. Hence, sensitivity analyses for modeling parameters were not thoroughly addressed in their study. 
In our study, we propose an integrated modeling framework combining traffic emission models and air pollutant dispersion models to estimate traffic emissions and dispersion across a large region. Unlike conventional approaches in which traffic simulation and meteorological models are jointly used to estimate traffic and meteorology data, this study employs various real world, real-time data sources in place of simulated data in order to best estimate traffic emissions and dispersion. Since it is not realistic and cost effective to install pollutant emission sensors at high density along all highways in a region, the proposed integrated modeling framework implements the concept of traffic sensors + meteorology sensors $=$ emission sensors to minimize cost. Combining data from traffic and meteorological sensors, which are relatively low-cost, effectively produces the information provided by emission sensors.

The remainder of this paper is organized as follows. An overview of the proposed framework will be introduced in the next section. Next, the emission and dispersion modeling process will be explained followed by the data description. After sensitivity analyses based on the proposed integrated modeling framework are presented, conclusions and future work will be covered in the final section.

\section{Integrated Modeling Framework}

The proposed integrated modeling framework consists of two major components, namely modeling methodology and data source integration. The proposed framework is designed to evaluate the magnitude of vehicle emissions and estimate pollution concentrations in a large region. Therefore, the modeling work can be further split into three models, including a traffic pollutant emission model, pollutant dispersion model and visualization model. The data source integration includes traffic data collected from Intelligent Transportation Systems (ITS) infrastructure, meteorological data and geographical data.

Figure 1 demonstrates the integrated framework, including all of the data sources and relevant models. Each major component will be explained in detail in the following sections.

\section{Modeling Methodology}

\section{Traffic Emission Modeling}

Several emission models have been developed worldwide to estimate traffic emissions. The World Road Association (PIARC), a non-profit organization, proposed the PIARC methodology to estimate vehicle emissions (PIARC, 2012). The European Environment Agency (EEA) has contributed to developing a software program, COPERT, which calculates air pollutants from roadways (Gkatzoflias et al., 2009). In the United States, the California Air Resources Board's Emission FACtors (EMFAC) model (EMFAC, 2007) and the National Cooperative Highway Research Program's Comprehensive Model Emission model (Barth et al., 2000) have been developed to calculate the emissions produced by cars, trucks and motorcycles.

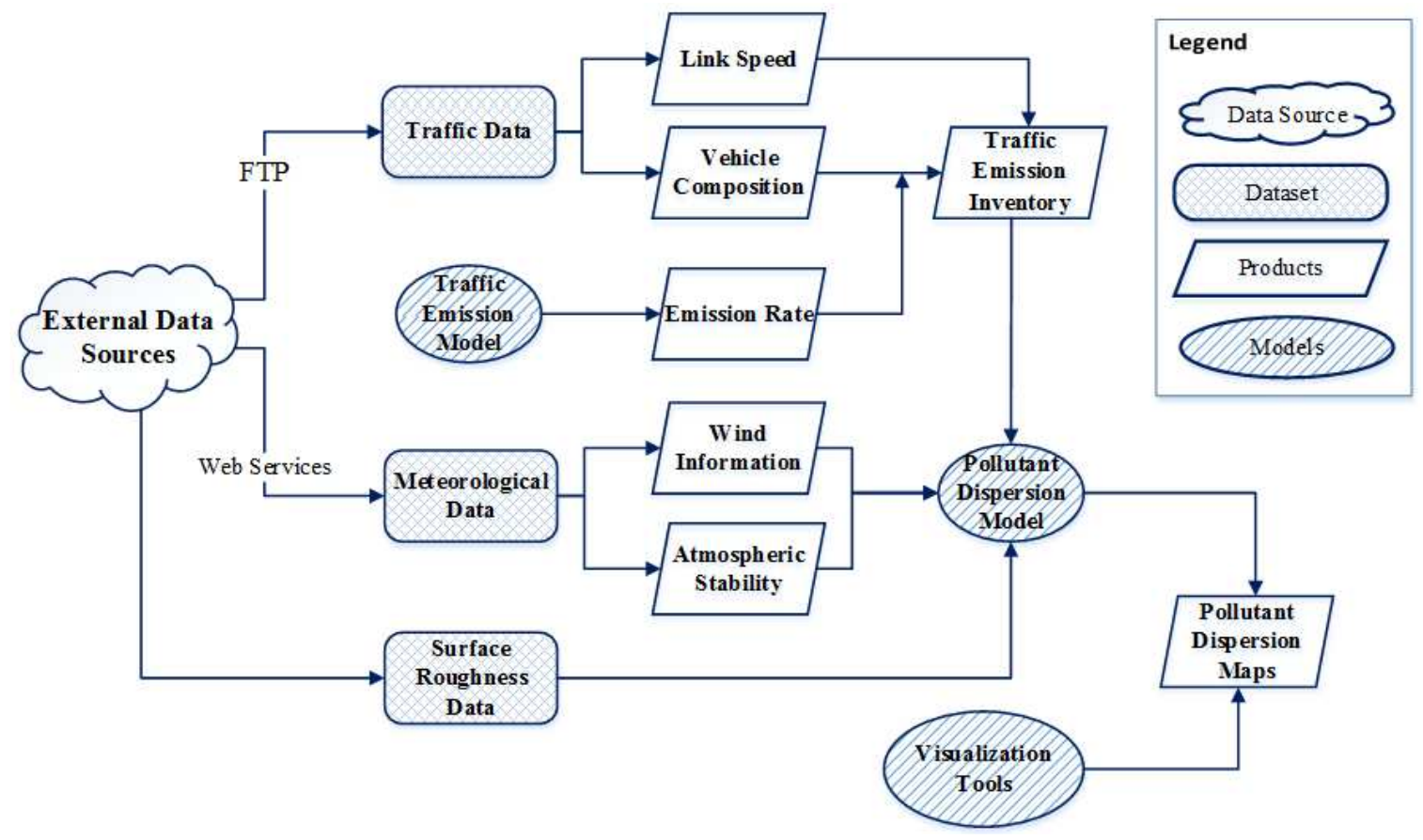

Fig. 1. Integrated framework for traffic pollutant emission and dispersion modeling 
The US Environmental Protection Agency (EPA) has developed the MOBILE model for estimating emissions of hydrocarbons, $\mathrm{CO}$ and other pollutants from vehicles. Recently, the MOtor Vehicle Emissions Simulator (MOVES, 2011) model has been developed in place of MOBILE. The EPA recommends using the new traffic emission model, MOVES, as opposed to others (e.g., MOBILE) to quantify the air pollution caused by vehicles. The EPA believes MOVES can predict a much more comprehensive range of pollutants. Three types of vehicle movement built into MOVES are used to estimate traffic emissions using: (a) Roadway link average speed; (b) link driving schedule; and (c) detailed vehicle operating mode. Our study used option (a) because of the ITS data availability and the suitability for a large network (Lee et al., 2012).

A two-step approach is used to estimate the total traffic emission inventory. The first step is to collect link speed and volume data from the ITS traffic detectors. The second step is to estimate the emission rate using MOVES model. These two steps are detailed in the following sections.

\section{Link Speed and Volume Data Collection}

A link is defined as a roadway segment bounded by two consecutive traffic sensors. The length of a particular link is a fixed value. The upstream and downstream traffic sensors on the link collect speed data. Link speeds can be estimated using the average of upstream and downstream speed. The instantaneous travel time estimation model ( $\mathrm{Li}$ et al., 2006) refers to the average speeds as the link speeds. Assuming "vehicles passing the upstream traffic sensor travel with the average speed," (Li et al., 2006) the traffic volume data from the upstream traffic sensor is used to represent the traffic volume on each of the links.

\section{Emission Rate Estimation}

Three essential variables used for generating emission rates in the MOVES model are (a) roadway type, (b) pollutant type and (c) traffic stream composition. Roadway and pollutant types are static variables but vehicle composition is a dynamic variable that is assumed to vary with time. Determining vehicle composition is critical because different types of vehicle release different amounts of pollutants. Vehicle types in the MOVES model are predefined according to the Federal Highway Administration's (FHWA) classification schema (where 13 vehicle types are classified based on size and number of axles). Due to the data collection limitation, the vehicle classification information is temporarily not available in this study, but the data can be easily incorporated into the current framework once the classification data is ready. In this study, static vehicle composition information provided by the Missouri Office of Administration's annual report was used (SMOA, 2013). Seven vehicle types defined in the vehicle composition information report are mapped to the FHWA classification schema. Each vehicle type, stated as type $e_{m}$, is assigned with a fixed value of percentage, $\left.p\right|_{t y p e_{i}}$. Therefore, the number of vehicles by type on a link $i$ can be calculated as $\left.v_{i} * p\right|_{t y p e_{m}}$, where $v_{i}$ represents the volume on link $i$.

In order to estimate the emission rates $(r)$, scenarios are created by joint use of vehicle types, unit traveled miles and single vehicle. The resulting traffic emission rates given vehicle types (type $e_{m}$ ) and corresponding speed $\left(s_{n}\right)$ are expressed as $\left.r\right|_{t y p e_{m}, s_{n}}$ with the unit "per mile per vehicle." "These values include the rates for exhaust and evaporative emissions that occur while vehicles are on real roads" (MOVES, 2011).

\section{Traffic Emission Inventory Calculation}

Given a specific vehicle type and link speed, an emission inventory on a link is the product of emission rate, traveling distances and the number of vehicles. Equation 1 and 2 formulate the traffic emission inventory calculation.

$$
\begin{aligned}
& \left.E I_{i}\right|_{\text {type }_{m}, s_{n}}=\left.\left.L_{i} * v_{i} * p\right|_{\text {type }_{m}} * r\right|_{\text {type }_{m}, s_{n}} \\
& \left.E I_{i}\right|_{s_{n}}=\left.\sum_{m=1}^{7} E I_{i}\right|_{\text {type }_{m}, s_{n}}
\end{aligned}
$$

Where:

$$
\begin{aligned}
\left.E I_{i}\right|_{t y p e_{m}, s_{n}}= & \text { The traffic emission inventory on link } i, \\
& \text { given a specific vehicle type type } e_{m} \text { and } \\
& \text { speed } s_{n} \\
= & \text { The number of vehicles running on the link; } \\
v_{i} & \text { The percentage of vehicles by types } \\
\left.p\right|_{\text {type }_{m}}= & \text { The emission rate given a specific vehicle } \\
\left.r\right|_{t y p e_{m}, s_{n}}= & \text { type type } e_{m} \text { and speed } s_{n} \\
\left.E I_{i}\right|_{s_{n}}= & \text { The traffic emission inventory on link } i, \\
& \text { given a specific vehicle speed } s_{n}
\end{aligned}
$$

\section{Emission Dispersion Modeling}

As described in the Introduction section, previous research (Amirjamshidi et al., 2013), used both total traffic emissions and air dispersion models to estimate pollutant dispersion with wind velocity and wind direction information. Our study includes two additional essential factors in the modeling process: Atmospheric stability and surface roughness. These 
two factors have previously been ignored when modeling emission dispersion.

\section{Traffic Pollutant Dispersion Estimation}

Traffic pollutant dispersion estimation begins with estimating the magnitude of traffic emissions. The emission amount is then coupled with atmospheric conditions (e.g., wind speed and direction and atmospheric stability) to estimate the pollutant concentration at various locations. Several traffic pollutant dispersion models, such as AERMOD and CALPUFF, have been developed to estimate pollution from point sources, area sources and volume sources. Since traffic is a type of line pollution source, this study used the line-based air pollutant dispersion model, CALINE3, (Benson, 1979) to estimate pollutant concentrations because it is designed to estimate the concentrations of freeway traffic pollutants. Moreover, the EPA describes the advantages of CALINE3 by stating that “... this steady-state Gaussian model can be applied to determine air pollution concentrations at receptor locations downwind of 'at-grade,' 'fill,' 'bridge,' and 'cut section' highways located in relatively uncomplicated terrain."

Many parameters in the CALINE3 model determine the pollutant concentration at receptors (individual monitoring sites). For example, "averaging time" acts as a temporal attribute but also affects the pollutant concentration. This occurs because the distribution of pollutants tends to change rapidly depending on atmospheric conditions including wind velocity and direction. Besides the temporal parameter, the pollutant concentration of a receptor is also determined by the emission inventory of pollutants, the location of the receptor, wind velocity, wind direction, surface roughness and the degree of atmospheric stability.

Most of the information used in this study is geographically continuous, such as surface roughness, wind direction and pollutant concentration. To increase computational performance and improve visualization, the continuous surface of the study region, the Greater St. Louis area, was discretized into a $150 * 150$ matrix (equivalent to 22,500 unit areas). The center point of each unit area was used as the location of the receptor. The CALINE3 model was applied to each unit area to compute the traffic pollutant concentration. The pollutant concentration in a unit area could be generated by multiple roadways. Therefore, the total pollutant concentration of a unit area is the sum of the pollutant concentration from surrounding highways. Equation 3 and 4 present the traffic pollutant dispersion estimation process.

$$
\left.P C U(\text { unit area })\right|_{\text {link } i}=C A L I N E 3
$$

$P C U($ unit area $)=\sum_{i=1}^{N} P C C_{\text {link } i}$

Where:

$P C C_{\mid \text {link } i}=$ The pollutant concentration of a unit area caused by running vehicle on link $i$

PCC = The accumulated pollutant concentration based on individual links

\section{Atmospheric Stability Measurement}

As stated in the Introduction section, only a limited amount of previous research addressed atmospheric stability in the air pollutant dispersion modeling process. Because meteorological data is widely available, atmospheric stability can be measured. Turner (1994) recommended different methods to measure atmospheric stability, such as Pasquill-Gifford stability categories based primarily on solar radiation, surface wind velocity and direction. Wind velocity and direction are observed and monitored by meteorology sensors in many locations. However, solar radiation information was unavailable in our study. Because of this, our study adopted the atmospheric stability classification system used by the Nuclear Regulatory Commission (Slade, 1968). In this system, atmospheric stability classes are based on the standard deviation of horizontal wind direction. Table 1 shows the atmospheric stability classes corresponding to the standard deviation range of horizontal wind direction. Six atmospheric stability classes are defined on the basis of horizontal wind direction rate of change. Stability Class A refers to the most unstable condition while Stability Class $G$ refers to the most stable atmospheric condition.

The standard deviation of wind direction can be calculated using Equation 5.

$\sigma_{\theta}=\sqrt{\frac{1}{N} * \sum_{i=1}^{N}\left(w d_{i}-\mu\right)^{2}}$

Where:

$\sigma_{\theta}=$ The standard deviation of the observed horizontal wind direction in a time period

$w d_{i}=$ The individual observed horizontal wind direction (in degrees)

$N=$ The number of observed $w d_{i}$ in a time period

$\mu=$ The average value of the $\mathrm{N}$ observed $w d_{i}$

\section{Surface Roughness}

The CALINE3 user's guide (Benson, 1979) was referenced in order to determine the value of surface roughness. Table 2 lists the recommended values of surface roughness depending on the surface type. 
Table 1. Atmospheric stability class with the range of standard deviation of wind direction

\begin{tabular}{ll}
\hline Stability class & Standard deviation of horizontal wind direction (degree) \\
\hline A & $\sigma_{\theta} \in(22.5,+\infty)$ \\
B & $\sigma_{\theta} \in(17.5,22.5]$ \\
C & $\sigma_{\theta} \in(22.5,17.5]$ \\
D & $\sigma_{\theta} \in(7.5,12.5]$ \\
E & $\sigma_{\theta} \in(3.75,7.5]$ \\
F & $\sigma_{\theta} \in(2.0,3.75]$ \\
G & $\sigma_{\theta} \in(0,2.0]$ \\
\hline
\end{tabular}

Table 2. Surface roughness for different land use (excerpt from page 12 (Benson, 1979))

\begin{tabular}{ll}
\hline Type of surface & Surface roughness values $(\mathrm{cm})$ \\
\hline Smooth mud flats & 0.001 \\
Tarmac (pavement) & 0.002 \\
Dry lake bed & 0.003 \\
Smooth desert & 0.030 \\
Grass $(5-6 \mathrm{~cm})$ & 0.750 \\
Grass $(4 \mathrm{~cm})$ & 0.140 \\
Alfalfa $(15.2 \mathrm{~cm})$ & 2.720 \\
Grass $(60-70 \mathrm{~cm})$ & 11.40 \\
Wheat $(60 \mathrm{~cm})$ & 22.00 \\
Corn $(220 \mathrm{~cm})$ & 74.00 \\
Citrus orchard & 198.0 \\
Fir forest & 283.0 \\
Single family residential & 108.0 \\
Apartment residential & 370.0 \\
Office & 175.0 \\
Central business district & 321.0 \\
Park & 127.0 \\
\hline
\end{tabular}

\section{Study Data}

Model implementation and emission estimates are highly dependent upon data availability. Unlike conventional approaches, data used in this study was collected from public instead of propriety sources. Since the data was publicly available to users, one can expect that the applicability of the proposed integrated framework would be increased.

\section{Traffic Data}

Major freeways and highways in the Greater St. Louis area were covered by over 1000 traffic sensors operated by the Missouri Department of Transportation (MoDOT). These traffic sensors monitored traffic conditions in the region for traffic operations purposes. They were generally spaced one mile apart on roadways. Figure 2 depicts the sensor locations in the region. Three fundamental traffic parameters-volume, speed and occupancy (percentage of time the detector is occupied by passing vehicles)-had been collected every $30 \mathrm{sec}$ and sent to the University of Arizona Smart Transportation Laboratory through a File Transfer Protocol (FTP) server.

\section{Open Data}

Open data is a relatively new concept of data acquisition. Open data is defined as data that can be freely accessed by the public and reused and redistributed by anyone without requesting copyright permissions (OKF, 2013). The meteorological and surface roughness data used in this study were open data.

\section{Meteorological Data}

Meteorological data can be obtained from meteorological sensors. Wunderground (2014) provided meteorological data to the public and managed a variety of meteorological sensors. The sensors covered the entire greater St. Louis area and the unit of measured area was based on zip code. The large coverage area of the meteorological sensors made pollutant dispersion estimation over a large region feasible. The meteorological information included wind velocity, wind direction and sun radiation, all updated every five minutes.

\section{Surface Roughness Data}

Surface roughness is not constant across a large region. Since the study region was discretized into $150 * 150(22,500)$ unit areas, surface roughness information had to be manually collected by interpreting satellite images provided by Google Earth. Since land use information (e.g., apartment, residential and parks) rarely changes over short time periods, the surface 
roughness data from the images was considered to be essentially static information.

\section{Traffic Pollution Dispersion and Sensitivity Analysis}

This section describes the concept of traffic sensors + meteorology sensors = emission sensors using the proposed integrated modeling framework. It should be noted that every possible pollutant in existence (e.g., $\mathrm{CO}_{2}$, NOx and PM) were incorporated into the modeling framework, but only $\mathrm{CO}$ was used to demonstrate the performance of the proposed framework. Traffic pollution dispersion maps are presented first to demonstrate how pollution concentration varies over time and two sensitivity analyses on atmospheric stability and surface roughness are then presented because these two factors were not addressed in previous studies.

\section{Traffic Pollution Dispersion Maps}

Figure 3 shows three traffic pollution dispersion maps using meteorological data from May 6, August 15 and October 24, 2013. The wind velocities and directions during the three days were significantly different; the wind on May 6, 2013 was fairly light with an average wind velocity of $1 \mathrm{~m} \mathrm{sec}^{-1}$ from the north and Class $G$ atmospheric stability; the wind on the other two days was relatively strong and atmospherically unstable. As Fig. 3 shows, (1) pollution were more concentrated along roadways when the wind was light; and (2) pollution was dispersed along the wind direction in Fig. 3b, while, the pollution were dispersed on both sides of the roadways when the atmosphere was unstable in Fig. 3c.

\section{Sensitivity Analysis: Atmospheric Stability}

Atmospheric stability plays an important role in estimating air pollutant dispersion. To evaluate the impact of atmospheric stability on traffic pollutant dispersion, two scenarios were developed. Both scenarios used data collected on July 11, 2012 from 4-5 pm. Scenario 1 used the most stable atmosphere condition (Class G), while Scenario 2 used the most unstable atmosphere condition (Class A). Figure 4 presents the traffic pollutant emission and dispersion patterns for these two scenarios.

For both Fig. 4a and 4b, traffic pollutants were concentrated along the roadways, especially around interchanges. The polluted areas in Fig. $4 \mathrm{~b}$ dispersed more widely due to the unstable atmospheric condition. It was found that 7049 unit areas in Fig. 4b were polluted with a concentration greater than $42 / \mathrm{m}^{3}$ in contrast to 6219 unit areas in Fig. 4a. The polluted region increased by approximately $13.3 \%$ due to the decline of atmospheric stability.

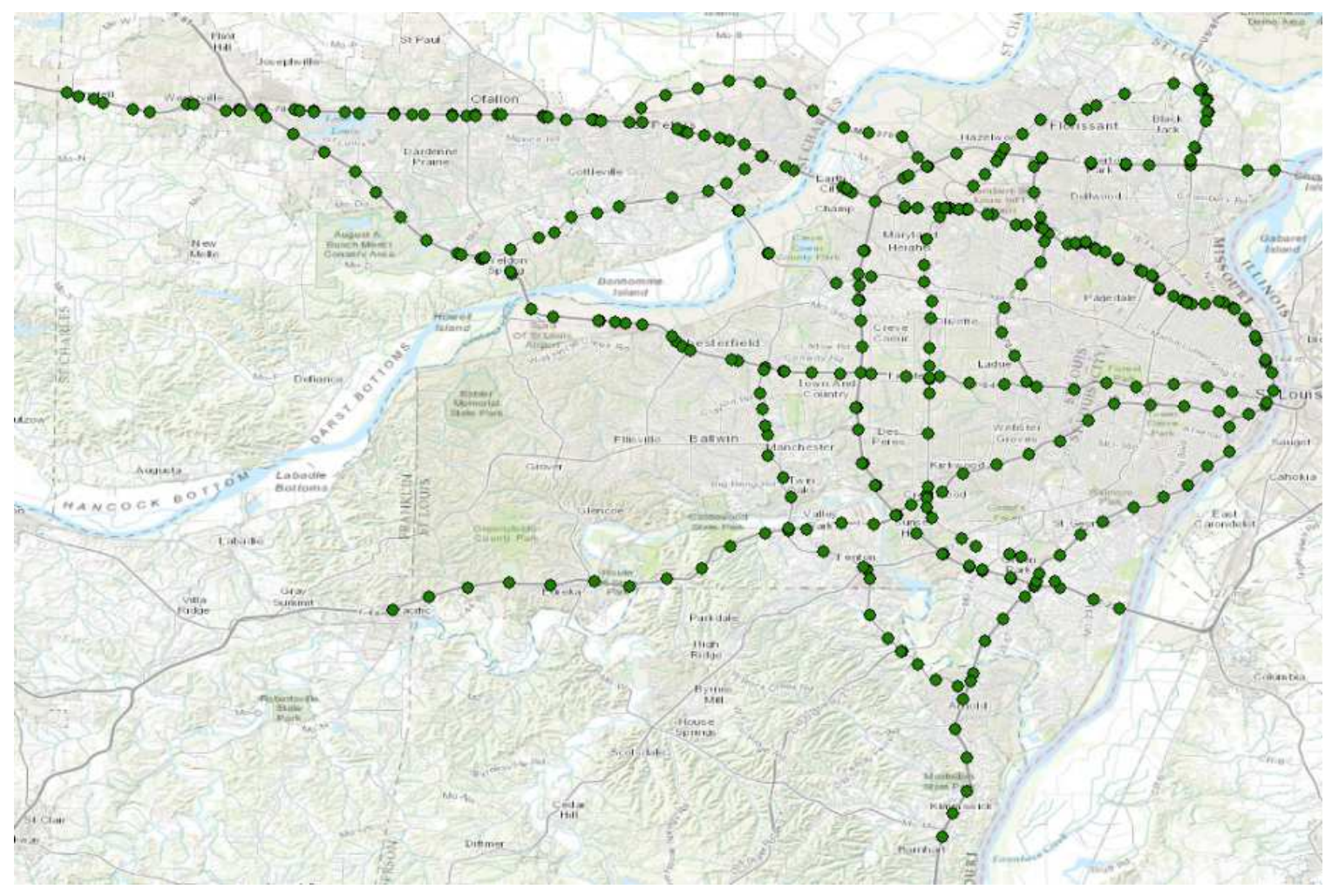

Fig. 2. Locations of traffic sensors in the Greater St. Louis area (Background image is from ArcGIS Online World Topographic Map) 


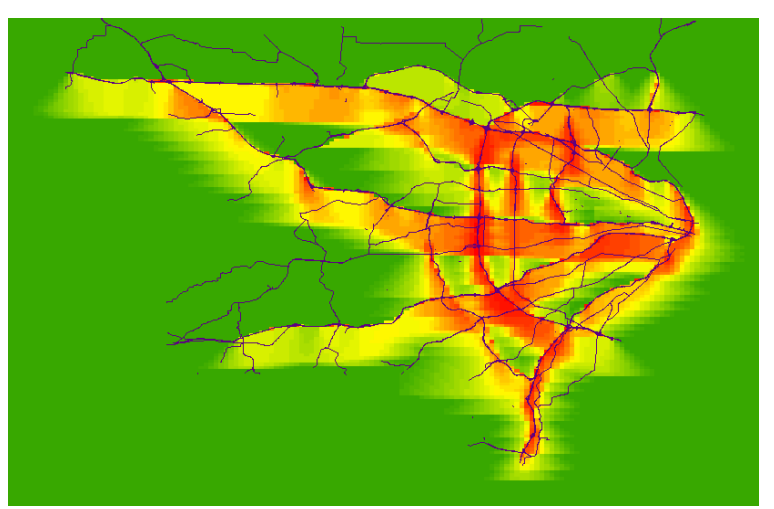

(a)

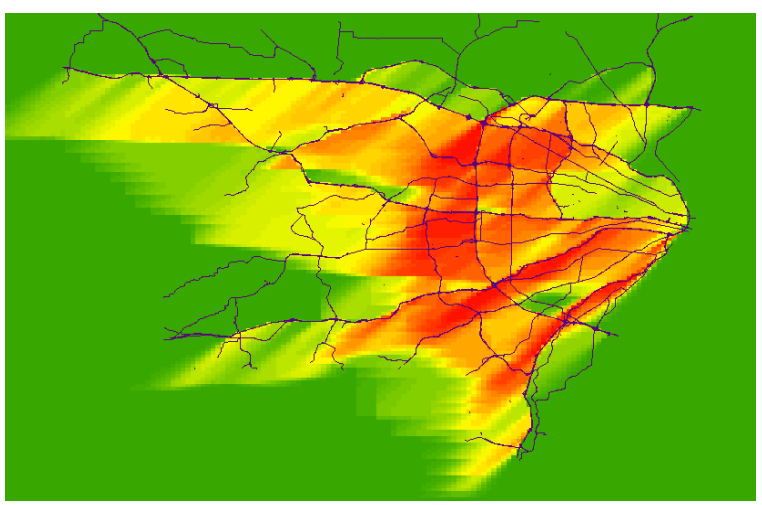

(b)

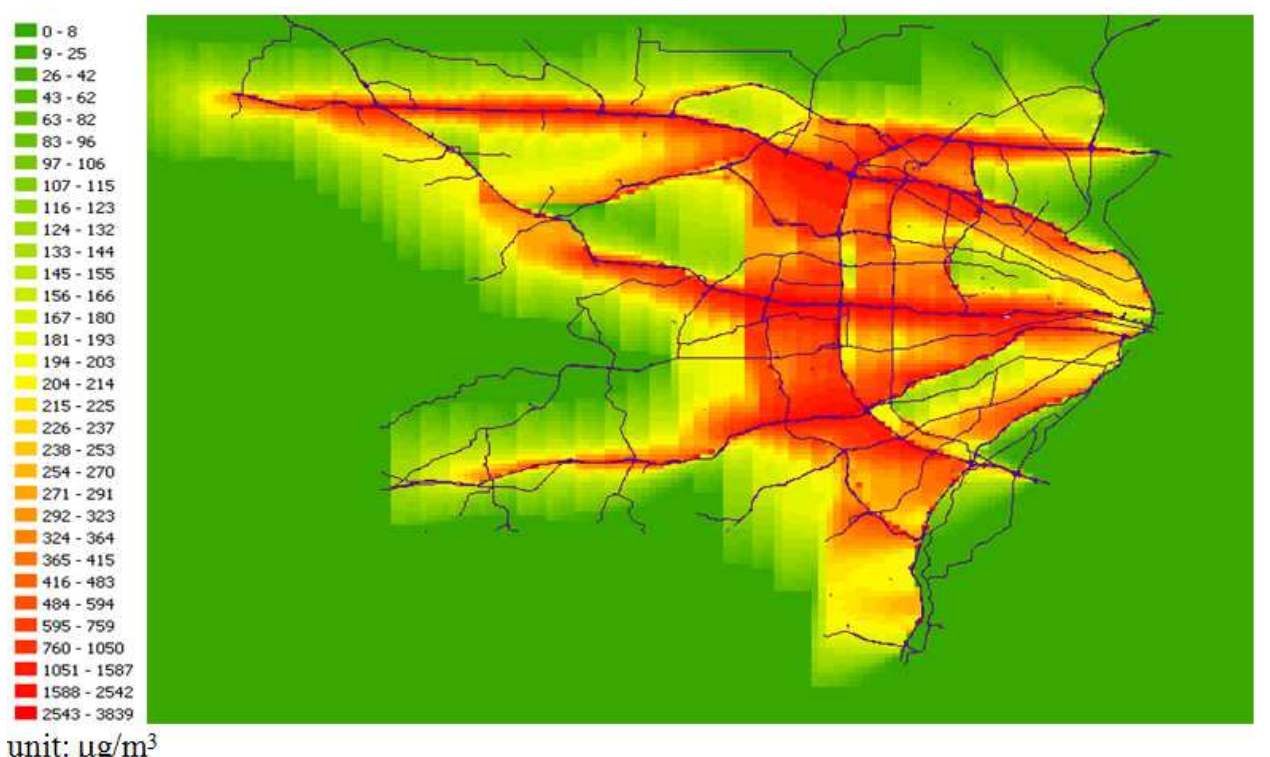

(c)

Fig. 3. Pollution dispersion maps in the greater St. Louis area (a) Atmospheric stability: Class G (the most stable case); wind velocity: $1 \mathrm{~m} / \mathrm{s}$; wind direction: north; 1-2 pm on May 6 (b) Atmospheric stability: Class C; wind velocity: $4 \mathrm{~m} / \mathrm{s}$; wind direction: northeast; 1-2 pm on Aug. 15, 2013 (c) Atmospheric stability: Class A (the most unstable case); wind velocity: $5 \mathrm{~m} / \mathrm{s}$; wind direction: north; $2-3 \mathrm{pm}$ on Oct. 24,2013

It was also found that the less-concentrated traffic pollutants were more evenly dispersed in the unstable atmosphere condition. To compare the difference, two areas of interest, Area A and Area B, are marked in Fig. 4. Area $\mathrm{A}$ was a residential area surrounded by Interstate 70 and Interstate 170, whereas Area B was a residential area that was surrounded by more freeways (i.e., Interstate 44, Interstate 64 and Interstate 270). Table 3 shows the air pollutant statistics in the two areas. As expected, Area A was impacted by traffic pollutant because this area was surrounded by freeways. The average pollutant concentration in the two areas under the stable condition was 270.1 and $489.2 \mu \mathrm{g} / \mathrm{m}^{3}$, respectively. The average concentrations under the unstable atmosphere condition were decreased by 15.0 and $5.0 \%$, respectively. Therefore, the traffic pollutants dispersed more when the atmospheric condition became unstable. Atmospheric stability had a higher impact on Area A (the area with low pollutant concentration). The result was consistent with results by Snow (2002), which showed that traffic pollutants would be trapped in the neighborhoods along roadways when the atmospheric condition is stable.

Moreover, atmospheric stability had a much greater impact on the standard deviation of pollutant concentration in Area B. The standard deviation decreased by $60.2 \%$ due to atmospheric instability, indicating that pollutants dispersed faster when the concentration was high and atmospheric stability was low. It is worth mentioning that due to relatively large analysis cells and the effects of surface roughness, the colors representing the pollution concentrations in both Fig. 3 and 4 may be visually discontinuous. 

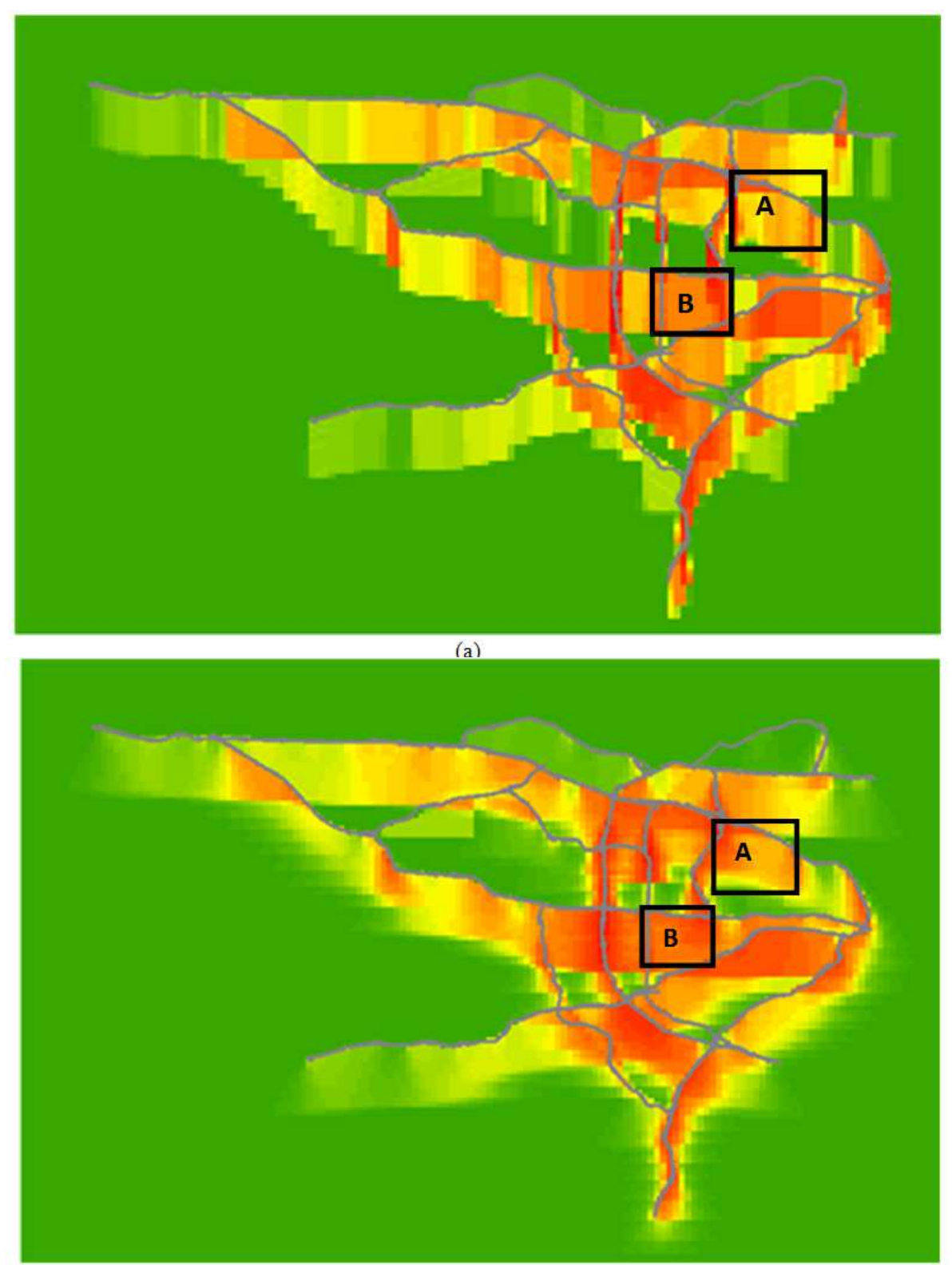

(b)
0.8
$9-25$
- $26-42$
- $43-62$
- $63-82$
드 83-96
- $97-106$
$107-115$
미 116-123
밈 $124-132$
$133-144$
미 $145-155$
$156-166$
$167-180$
$181-193$
$194-203$
$204-214$
- $215-225$
$226-237$
$238-253$
다- 254
미 - 291
- $292-323$
피 $324-364$
- $365-415$
때 $416-483$
$484-594$
- $595-759$
- $760-1050$
$1051-1587$
1588-2542
-2543-3839
unit: $\mu \mathrm{g} / \mathrm{m}^{3}$

Fig. 4. Atmospheric stability sensitivity analysis (a) Atmospheric stability: Class G (the most stable case); wind velocity: $2 \mathrm{~m} / \mathrm{s}$; wind direction: north; 4-5 pm on July 11, 2012 (b) Atmospheric stability: Class A (the most unstable case); wind velocity: $2 \mathrm{~m} / \mathrm{s}$; wind direction: north; 4-5 pm on July 11, 2012

\section{Sensitivity Analysis: Surface Roughness}

Pollutants disperse differently on surfaces with different roughness. Three scenarios were created to help understand the impacts of surface roughness on pollutant dispersion. Forest Park was selected as the area of interest (with an area of $5.55 \mathrm{~km}^{2}$ ) because it was a major recreational green area in St. Louis:

- Scenario A (Baseline): The surface roughness remains as park type (roughness value: 127)
- Scenario B (Pavement): Forest Park covered by pavement (roughness value: 0.002 )

- Scenario C (Fir Forest): Forest Park covered by fir trees (roughness value: 283 )

As shown in Table 4, the average pollutant concentration was highest in Scenario $\mathrm{C}$ and lowest in Scenario B. These results indicated that the fir forest can limit pollutant dispersion and capture more pollutants in the area. In contrast, when no park exists in the area, the pollutants would be more efficiently dispersed. 
Table 3. Pollutant statistics comparison

\begin{tabular}{|c|c|c|c|c|c|c|}
\hline \multirow[b]{2}{*}{ Atmospheric stability } & \multicolumn{3}{|l|}{ Area A } & \multicolumn{3}{|l|}{ Area B } \\
\hline & Stable & Unstable & Decreased by & Stable & Unstable & Decreased by \\
\hline Average pollutant concentration $\left(\mu \mathrm{g} / \mathrm{m}^{3}\right)$ & 270.1 & 229.5 & $15.0 \%$ & 489.2 & 464.9 & $5.0 \%$ \\
\hline Standard deviation of pollutant concentration $\left(\mu \mathrm{g} / \mathrm{m}^{3}\right)$ & 141.9 & 125.2 & $11.8 \%$ & 295.6 & 117.6 & $60.2 \%$ \\
\hline
\end{tabular}

Table 4. Pollutant concentration comparison between scenarios

\begin{tabular}{lll}
\hline Scenario & Average pollutant concentration $\left(\mu \mathrm{g} / \mathrm{m}^{3}\right)$ & Increase/Decrease \\
\hline A (Baseline) & 377.8 & - \\
B (Pavement) & 370.4 & $-2.0 \%$ \\
C (Fir Forest) & 379.4 & $+0.4 \%$ \\
\hline
\end{tabular}

\section{Conclusion}

Air quality is of great concern to the public and managing agencies. Pollutants, such as $\mathrm{CO}, \mathrm{CO}_{2}$ and $\mathrm{PM}$, can negatively impact individual health. Vehicular exhaust is a major source of these pollutants. In order to quantify vehicle pollutants, a considerable amount of previous research has contributed to modeling traffic pollutant emission. Computational simulation-based methods have been popular due to the limited availability of traffic and meteorological data. The simulation would become technically not flexible when study areas are relatively large.

Since traffic and meteorological data has recently become much more available, this study proposed the concept of traffic sensors + meteorology sensors $=$ emission sensors to estimate traffic pollutant emissions over a large area. The concept was proposed to combine real world data from traffic and meteorology sensors to technically compensate for the limited availability of emission sensors in a large area. An integrated modeling framework was therefore proposed to implement the concept. The framework incorporated conventional traffic and meteorological data to estimate traffic pollutant emission and dispersion over a large area. Two primary existing emission-related models were included in the framework: The MOVES model and CALINE3 model. The MOVES model was used to generate the emission rates based on average vehicle speed on freeways. Then, the total amount of pollutant emissions was estimated by incorporating freeway traffic data and the emission rates from the MOVES model. The CALINE3 air pollutant dispersion model was used to estimate pollutant dispersion along a regional freeway network. Rather than using conventional simulationbased approaches to obtain traffic and meteorological data, this study utilized the real world data. This data (including wind direction and surface roughness) and the calculated pollutant emissions were streamed into the CALINE3 model as inputs.

The results from the proposed traffic pollutant emission estimation and dispersion framework are summarized below:
- Typically, primary inputs for conventional simulation-based methods of estimating vehicle emissions have included Origin-Destination (O-D) traffic information. Detailed vehicle movements on roadways would be simulated and then vehicle emissions could be estimated using these movements. Our study used real world traffic data instead, along with meteorological and geographical data. Using these inputs and relevant emissions models, traffic pollutant emissions could be more accurately estimated than by simply using conventional simulation-based methods

- Pollutant emissions caused by traffic were estimated in the Greater St. Louis area using the proposed framework. It is worth mentioning that the proposed framework was designed for roadway vehicle emissions, which are a major pollution source. However, the overall pollution in the Greater St. Louis area could be estimated by considering other pollution sources (e.g., pollution from industries). Future work could focus on integrating other pollution sources into the current framework in order to estimate overall pollution across a large region

- One of the major products from the framework, the pollutant dispersion map, has many potential applications. The sensitivity analyses demonstrated the applicability of this framework by implementing the three scenarios. The results of the sensitivity analyses clearly indicated that the forests can limit pollutant dispersion and retain more pollutants. Therefore, land with different surface roughness values can affect pollutant dispersion. These results could be useful for future land-use planning and guidelines, if a set of emission dispersion maps could be produced using yearlong traffic and meteorological data

- The pollutant dispersion maps also showed areas where the public would be most exposed to traffic pollutants

- Overall, the integrated platform provides many possibilities for applications in inter-disciplinary research. To further improve the framework, future work could focus on investigating different air 
pollutant dispersion models and evaluating the interactions between different pollutants. Furthermore, the framework could be implemented in a system to monitor real-time pollutant dispersion or predict future pollutant dispersion trends

\section{Acknowledgement}

The authors would like to acknowledge the funding support furnished by the Center for Sustainability at Saint Louis University. We also appreciate the data support provided by the Missouri Department of Transportation (MoDOT), U.S.

\section{Funding Information}

This study was funded by the Center for Sustainability at Saint Louis University, Saint Louis, Missouri, U.S.

\section{Author Contributions}

Shu Yang: Designed the research plan, participated in computer programming, data preparation and contributed to the paper writing.

Yao-Jan Wu: Organized the study and contributed to the paper writing.

John Woolschlager: Member of OneSTL project team and reviewed the manuscript.

\section{Ethics}

All of the authors have read and approved the manuscript and there are no ethical issues involved.

\section{References}

Aldrin, M. and I.H. Haff, 2005. Generalised additive modelling of air pollution, traffic volume and meteorology. Atmospheric Environ., 39: 2145-2155. DOI: 10.1016/j.atmosenv.2004.12.020

Amirjamshidi, G., T.S. Mostafa, A. Misra and M.J. Roorda, 2013. Integrated model for microsimulating vehicle emissions, pollutant dispersion and population exposure. Transport. Res., 18: 16-24.

DOI: $10.1016 /$ j.trd.2012.08.003

Barth, M., F. An, T. Younglove, G. Scora and C. Levine, 2000. NCHRP project 25-11 development of a comprehensive modal emission model.

Benson, P.E., 1979. Abridged user's guide for CALINE-3.

Berkowicz, R., M. Winther and M. Ketzel, 2006. Traffic pollution modelling and emission data. Environ. Modell. Software, 21: 454-460.

DOI: $10.1016 /$ j.envsoft.2004.06.013

CDPH, 2014. Traffic exhaust pollutants. California Department of Public Health.
EMFAC, 2007. Calculating emission inventories for vehicles in California.

Gkatzoflias, D., C. Kouridis, L. Ntziachristos and Z. Samaras, 2007. COPERT 4 computer programme to calculate emissions from road transport. Users Manual.

Hatzopoulou, M. and E.J. Miller, 2010. Linking an activity-based travel demand model with traffic emission and dispersion models: Transport's contribution to air pollution in Toronto. Transport. Res., 15: 315-325. DOI: 10.1016/j.trd.2010.03.007

Ishaque, M.M. and R.B. Noland, 2008. Simulated pedestrian travel and exposure to vehicle emissions. Transport. Res., 13: 27-46. DOI: $10.1016 /$ j.trd.2007.10.005

Jamriska, M., L. Morawska and K. Mergersen, 2008. The effect of temperature and humidity on size segregated traffic exhaust particle emissions. Atmospheric Environ., 42: 2369-2382. DOI: $10.1016 /$ j.atmosenv.2007.12.038

Jeng, S.T., K.S. Nesamani and S.G. Ritchie, 2013. A new approach to estimate vehicle emissions using inductive loop detector data. J. Intell. Transport. Syst., 17: 179-190. DOI: $10.1080 / 15472450.2012 .712495$

Jin, T. and L. Fu, 2005. Application of GIS to modified models of vehicle emission dispersion. Atmospheric Environ., 39: 6326-6333.

DOI: $10.1016 /$ j.atmosenv.2005.07.038

Lee, G., S. You, S.G. Ritchie, J.D. Saphores and R. Jayakrishnan et al., 2012. Assessing air quality and health benefits of the Clean Truck Program in the Alameda corridor, CA. Transport. Res., 46: 1177-1193. DOI: $10.1016 /$ j.tra.2012.05.005

Li, J.Q., W.B. Zhang and L. Zhang, 2009. A web-based support system for estimating and visualizing the emissions of diesel transit buses. Transport. Res., 14: 533-540. DOI: 10.1016/j.trd.2009.07.009

Li, R., G. Rose and M. Sarvi, 2006. Evaluation of speedbased travel time estimation models. J. Transport. Eng., 132: 540-547.

DOI: 10.1061/(ASCE)0733-947X(2006)132:7(540)

Ma, X., Y.J. Wu and Y. Wang, 2011. DRIVE Net: Escience transportation platform for data sharing, visualization, modeling and analysis. J. Transport. Res. Board, 2215: 37-49. DOI: 10.3141/2215-04

MOVES, 2011. Motor Vehicle Emission Simulator (MOVES) user guide for MOVES2010b.

PIARC, 2012. Road tunnels: Vehicle emissions and air demand for ventilation. PIARC Technical Committee 5 Road Tunnels.

Raaschou-Nielsen, O., O. Hertel, B.L. Thomsen and J.H. Olsen, 2001. Air pollution from traffic at the residence of children with cancer. Am. J. Epidemiol., 153: 433-43.

DOI: $10.1093 /$ aje/153.5.433 
Samaranayake, S., S. Glaser, D. Holstius, J. Monteil, K. Tracton et al., 2014. Real-time estimation of pollution emissions and dispersion from highway traffic. Comput. - Aided Civil Infrastruct. Eng., 29: 546-558. DOI: 10.1111/mice. 12078

Slade, D.H., 1968. Meteorology and atomic energy, TID-24190. U. S. Atomic Energy Commission, Washington, DC.

Smit, R., M. Poelman and J. Schrijver, 2008. Improved road traffic emission inventories by adding mean speed distributions. Atmospheric Environ., 42: 916-926. DOI: 10.1016/j.atmosenv.2007.10.026

Snow, D.A., 2002. Plant Engineer's Reference Book. 2nd Edn., Butterworth-Heinemann, Oxford, ISBN-10: 0750644524, pp: 862.

SMOA, 2013. State fleet management annual report. State of Missouri Office of Administration.
OKF, 2013. The Open Knowledge Foundation.

Turner, D.B., 1994. Workbook of Atmospheric Dispersion Estimates: An Introduction to Dispersion Modeling. 2nd Edn., CRC Press, ISBN-10: 156670023X, pp: 192.

Wu, Y.J., S. An, X. Ma and Y. Wang, 2011. Development of web-based analysis system for realtime decision support on arterial networks. J. Transport. Res. Board, 2215: 24-36. DOI: $10.3141 / 2215-03$

Wunderground, 2014. Retrieved from Wunderground.com

Xia, L. and Y. Shao, 2005. Modelling of traffic flow and air pollution emission with application to Hong Kong Island. Environ. Modell. Software, 20: 1175-1188. DOI: 10.1016/j.envsoft.2004.08.003 